\title{
EVALUATING ROUTE LEARNING PERFORMANCE OF OLDER AND YOUNGER ADULTS IN DIFFERENTLY-DESIGNED VIRTUAL ENVIRONMENTS: A TASK- DIFFERENTIAL ANALYSIS
}

\author{
I. E. Lokka*, A. Çöltekin \\ Department of Geography, University of Zurich, CH-8057, Zurich, Switzerland \{ismini-eleni.lokka, arzu.coltekin\}@geo.uzh.ch
}

\author{
Commission IV, WG IV/9
}

KEY WORDS: Navigation, ageing, task types, visual memory, spatial memory, virtual reality, realism, abstraction

\begin{abstract}
:
Navigating in unfamiliar environments is a complex task that requires considerable cognitive resources to memorize (and eventually learn) a route. In general, virtual environments (VEs) can be useful tools in training for route learning and improving route recall. However, the visual information presented in VEs, that is, what we choose to present in a virtual scene, can strongly affect the ability to recall a route. This is especially relevant when we consider individual differences, and people's varying abilities to navigate effectively. Taking various cognitive processes involved in route learning into account, we designed a multi-level experiment that examines route recall effectiveness in a navigation context. We conceptualized that the participants would have to recall information related to the route that is demanding on primarily visual, spatial, or visuospatial memory systems. Furthermore, because there is a clear link between memory capacity and ageing; we conducted our experiment with two different age groups (total 81 participants: 42 young people aged 20-30 yo and 39 older people aged 65-76 yo). We also measured participants' spatial abilities and visuospatial memory capacity for control purposes. After experiencing a pre-determined route in three different VEs (that we varied in levels of visual realism, and named as AbstractVE, MixedVE, and RealisticVE), each participant solved a list of tasks that was designed to measure visual, spatial, and visuospatial recall of the scene elements and information about the route. Participants solved these tasks immediately after experiencing the route in each VE, as well as after a week, thus we could measure 'learning' (delayed recall). Results from our study confirm the well-known decline in recall with age (young vs. older), provide new information regarding memorability of routes and VE scene elements over time (immediate $v s$. delayed), and most importantly demonstrate the crucial role the visual design decisions play in route learning and memorability of visuospatial displays.
\end{abstract}

\section{INTRODUCTION AND BACKGROUND}

Technological research programs dedicate considerable amount of resources to design virtual environments (VEs) as realistically as possible, because the goal is usually to simulate the real world in high-fidelity, that is, with as much detail as the computers can process/store, and as accurately as possible (Çöltekin \& Clarke, 2011). While such VEs are used in many interdisciplinary contexts with varying goals in mind; from the perspective of navigational research, VEs can be used both for understanding how people navigate, and assisting people to navigate more effectively. When we examine cognitive processes involved in human navigation, we see that there are various components that work separately and in combination. For example, to learn a route, one needs to process both visual and spatial input by encoding, storing, and retrieving the relevant information (Estes, 2014). These cognitive processes involved in navigation are affected by a number of different factors; such as the scene content (i.e., what is presented to the user), the task type (i.e., what is the goal of the user), and certain characteristics of the users (e.g., their expertise levels, their previous experiences in similar tasks, visuospatial abilities etc.).

In this study, we primarily examine the effects of task type (whether a task is predominantly demanding on visual, visuospatial or spatial memory systems) on route learning effectiveness, while we take ageing (age), and visual design of the VEs (visualization type), and the timing of when one needs to recall the route (recall stage) into account. Our main goal is to examine how visualization type affects route recall accuracy across these task types (i.e., visual, spatial and visuospatial recall) in the context of route learning with the different VEs we designed (AbstractVE, MixedVE, and RealisticVE), which primarily vary in levels of realism. When we designed these VEs, we primarily modified realism levels, because realism has been shown to be an important factor in cognitive load in various tasks involving visuospatial displays (Borkin et al., 2013; Smallman \& Cook, 2011), including route learning (e.g., \{Formatting Citation $\}$. Besides realism, it is well understood that highlighting is important for catching a viewer's attention, and attention is linked to memory (Cowan, 1995).

\section{METHODS}

For a user study that considers the factors described above; we created a fictitious city that we designed in three different ways:

1) a RealisticVE, representing the real world with high fidelity,

2) an AbstractVE with the least amount of photographic information where the location of the 
textured buildings (thus, highlighted using phototextures) are deliberately chosen, and

3) a MixedVE, in which we only highlighted selected structures using photo-realistic textures (Figure 1).

In the MixedVE, the amount and the location of the highlighted textures are carefully selected considering both visual realism and landmark theories (Röser, Hamburger, Krumnack, \& Knauff, 2012; Smallman \& Cook, 2011b). Specifically, we selected to highlight scene elements (using photo textures, in this case) in the MixedVE, only where the information should be relevant to route learning (further elaborated in Lokka \& Çöltekin, 2017). This 'relevance' was determined based on the findings in previous empirical work, for example, there is evidence that people pay attention to the scene elements at the intersection points, specifically to those at the direction of turn; and the structural elements, such as the road network may be important in route learning (e.g., Röser, Hamburger, Krumnack, \& Knauff, 2012). To achieve a broad understanding of our participants' route learning performance, we gave them an extensive list of tasks to solve, all of which related to recalling information. Specifically, participants were asked to identify

1) whether they have seen a particular scene on their route or not (which we considered to be primarily visual tasks),

2) which direction they turned at decision points (visuospatial tasks), and

3 ) whether they can reproduce a $2 D$ view of the route they viewed (a sketch); and if they could indicate cardinal directions by comparing orientation of beginning to ending point (spatial tasks).

Note that the classification of tasks into three primary categories (visual, spatial, visuospatial) is a rough classification, where the task primarily would require the use of spatial memory, visual memory or both. In reality, these memory systems possibly interact with each other constantly and in ways we cannot control (see Lokka \& Çöltekin, 2017 for further elaboration on our reasoning, and Cowan (1995) for an overview on memory systems). We examined participant performance separately for each task type, as well as at an aggregate level for total of tasks for each visualization type, and for each age group. Last but not least, route learning and navigational performance depends on one's spatial abilities and memory capacity, both of which decline with age (Park et al., 2002). Because these group differences (and in the context of this paper, especially aging) may impact the route learning performance, we examined two age groups: 25-35year-olds, and 65-75year-olds. Participants were told to recall the route that was shown to them (in all three VE types), and they solved the assigned tasks in two recall stages: immediately after experiencing the virtual route and approximately one week later again. In the delayed recall stage (one week later), they were not shown the VEs again, but were only given the recall tasks to solve. Complementary to findings from our previous studies (Lokka \& Çöltekin, 2017; Lokka, Çöltekin, et al., 2018), we present an overall analysis in this paper, examining all factors together. Note that results on spatial abilities and visuospatial memory abilities are not included in this analysis but presented at an aggregate level.

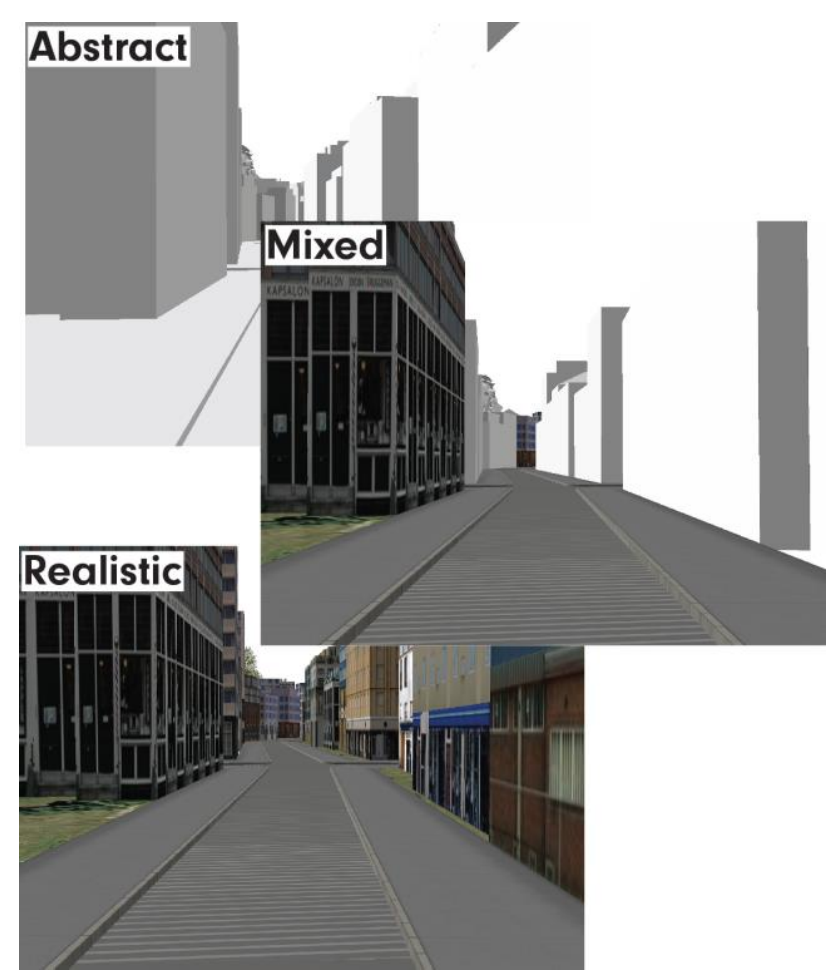

Figure 1. An illustration of the fictitious city designed in three different ways: an Abstract, a Mixed, and a Realistic VE.

\section{RESULTS}

Keeping the task type in the center of our examination, below we present how task types interact with all other factors in the experiment (age, visualization type, recall stage). First we evaluated the overall effects of the experimental tasks on the recall performance based on a 3 (task type) x 3 (visualization type) x 2 (age) x 2 (recall stage) mixed-design ANOVA. The results from the ANOVA revealed statistically significant differences for all factors (independent variables), apart from the task type, as seen in Figure 2. More specifically, we observed the following:

1) age, $\mathrm{F}(1,79)=23.54, \mathrm{p}<.001, \eta_{p}^{2}=.08$ (young: $62.3 \% \pm 25.7 \%$, older: $48.7 \% \pm 25.2 \%$ ),

2) recall stage $\mathrm{F}(1,79)=77.25, \mathrm{p}<.001, \eta_{p}^{2}=.03$ (immediate: $60.1 \% \pm 24.8 \%$, delayed: $51.4 \% \pm 27.2 \%$ ), and

3) visualization type $\mathrm{F}(2,158)=39.13 \mathrm{p}<.001, \eta_{p}^{2}=.02$ (AbstractVE: $53.1 \% \pm 26.6 \%$, MixedVE: $60.7 \% \pm$ $27.1 \%$, RealisticVE: $53.4 \% \pm 24.6 \%$ ) have statistically significant differences.

On the other hand, task type $\mathrm{F}(2,158)=1.02$, $\mathrm{p}>.05$ (visual task: $57.1 \% \pm 18.6 \%$, spatial task: $53.9 \% \pm 33.5 \%$, visuospatial task: $56.2 \% \pm 24.8 \%)$ did not lead to statistically significant differences. At this point, the findings suggest that the effects of the other three factors (age, recall stage and visualization type) are consistently present for all task types. We present a more indepth interaction analysis below. 
First, we conducted pairwise comparisons to examine the differences between the three VEs. This analysis revealed statistically significant differences between the MixedVE with both the Abstract $(\mathrm{p}<.001, \mathrm{~d}=0.28)$ and the Realistic VEs $(\mathrm{p}<.001, \mathrm{~d}=0.28)$. Thus we can see that participants' route recall accuracy is overall better with the MixedVE than the other two VEs, similar to our previous findings (e.g., see Lokka \& Çöltekin, 2017; Lokka, Çöltekin, et al., 2018).
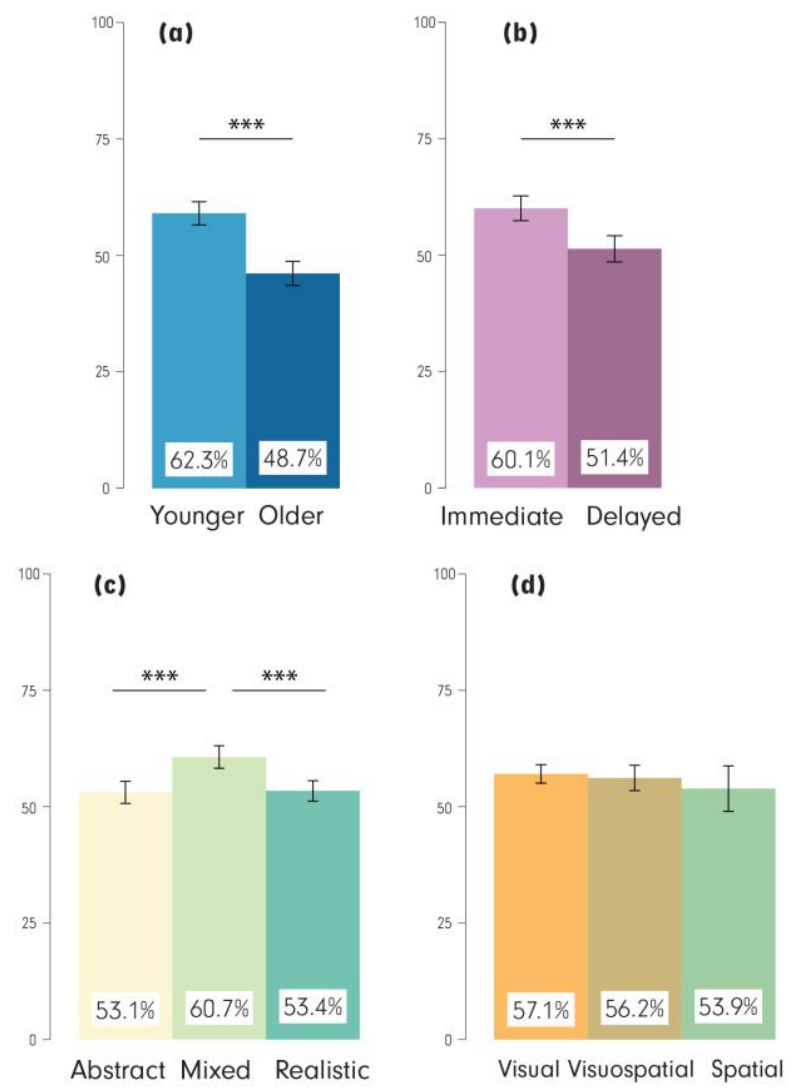

Figure 2. Main effects of the four independent variables: (a) age (younger/older), (b) recall stage (immediate/delayed), (c) visualization type (Abstract/Mixed/Realistic), and (d) task type (visual/visuospatial/spatial). $* * * \mathrm{p}<0.001$. Error bars: SEM.

In an effort to better understand the interactions, we examined the three task types by running separate mixed-design ANOVAs for all three tasks (as described in Lokka, Çöltekin, et al., 2018). In the following interaction analysis, we see that the performance patterns differ for the tasks (Figure 3). Specifically, for the visuospatial and spatial tasks, MixedVE facilitates the highest recall rates, which is followed by the Realistic and the AbstractVEs. For the visual task, however, the three visualization types have similarly low recall rates - close to chance levelthat probably do not allow the interactions to appear. Further interpretations are provided in the next section (Conclusions and Discussion).

For the visuospatial and spatial task types, we also see that there are statistically significant differences between the two age groups, whereas in the visual task, the differences are similarly low. On the other hand, for two of the task types (visuospatial and visual), the recall stage also yield statistically significant differences: Not surprisingly, participants recall the route overall better in the immediate recall stage than in the delayed recall stage. In comparing performance differences for the two recall stages, we see that the there are no performance differences for the spatial task(s), which is somewhat surprising, but possibly explained by active rehearsing of the sketch-drawing in the immediate recall stage for one of the spatial tasks.

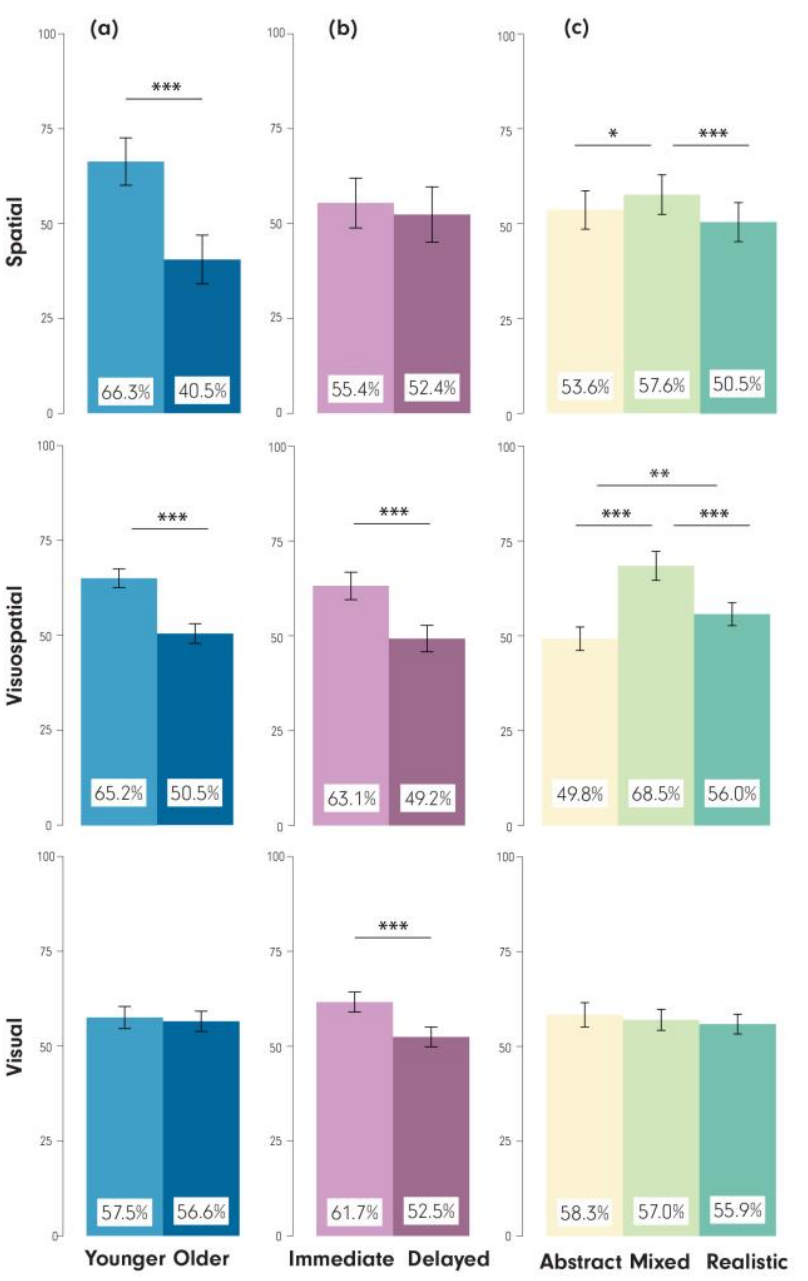

Figure 3. Main effects for (a) age (younger/older), (b) recall stage (immediate/delayed), and (c) visualization type (Abstract/Mixed/Realistic) for above: spatial, middle: visuospatial, below: visual tasks. ${ }^{* *} \mathrm{p}<0.001$. Error bars: SEM.

This figure is reprinted from Lokka, Çöltekin, et al., 2018.

To revisit the larger picture, we also report below that in the overall ANOVA, interactions between the factors below were statistically significant:

1) age $x$ visualization type $\mathrm{F}(2,158)=4.54, \mathrm{p}<.05, \eta_{p}^{2}=.00$,

2) age $x$ task type $(2,158)=11.36, \mathrm{p}<.001, \eta_{p}^{2}=.04$,

3) recall stage $x$ task $\mathrm{F}(2,158)=9.07, \mathrm{p}<.001, \eta_{p}^{2}=.01$,

4) visualization type $x$ task type $\mathrm{F}(4,316)=17.44, \mathrm{p}<.001$, $\eta_{p}^{2}=.03$, as well as 
5) recall stage $x$ visualization type $x$ task type $\mathrm{F}(4,316)=$ $8.08, \mathrm{p}<.001, \eta_{p}^{2}=.01$.

\section{CONCLUSIONS AND DISCUSSION}

Based on these results, we see that younger participants overall do better than older; all participants overall do better in the immediate recall stage than in the delayed recall stage; and the visualization type does affect the route recall accuracy, where the MixedVE overall offers advantages. These findings have been reported in our previous papers (e.g., Lokka \& Çöltekin, 2017; Lokka, Çöltekin, et al., 2018).

What we also see is that these results appear to apply to all task types examined at the aggregate level. A deeper analysis of interactions suggest, however, that some tasks are perhaps easier or harder with some visualization types (i.e., task type $x$ visualization type interact), and some tasks may be easier in one of the stages, for example, with one of the visualization types (i.e., recall stage $x$ visualization type $x$ task type). The separate ANOVAs for each task confirm the variability in the different patterns according to the task type.

The fact that the visual tasks do not display all the effects that we observe for the others might be because the responses were at the chance level. Alternatively, this might happen perhaps because for visual tasks, the memory is not affected by the realism levels as much as the spatial tasks. This remains to be tested in a dedicated experiment in the future. We also do not see an age difference in visual tasks. This may be plausible, as there is some evidence in the literature that the visual memory is 'spared' better with aging (Sekuler, Kahana, et al., 2005), suggesting that purely visual tasks may indeed be different than the tasks that have spatial components.

On the other hand, for purely spatial tasks, our findings did not suggest a difference between immediate and delayed recall stages, which is somewhat surprising. We believe this may be at least partly because of one of the tasks. Among what we considered 'spatial' task types, we asked the participants to sketch their route on an aerial view (2D printout of the VEs from the top). This drawing may have allowed them to learn the routes better and thus remember the $2 \mathrm{D}$ route also well a week later. If this was the case, it would reflect on the overall performance with spatial tasks. A deeper examination of this finding would also be possible with future experiments that are designed to test how the active sketching exercises affect long term spatial memory.

Overall, these findings confirm the central hypothesis that the way we design VEs has a strong effect on the way people work with them for a variety of tasks (also see Çöltekin, Lokka, \& Zahner (2016) for a brief literature review on related topics). We see that when people use differently-designed VEs to learn a route, memory is indeed directly affected by the visual information presented in the VEs. The fact that the visualization design affects people's recall performance for all tasks one way or another is a strong indication that we must pay attention to what we include in a virtual scene that is used in route recall experiments and applications.

The finding described above is especially important for navigational studies, because many navigation related experiments use VEs that are designed "ad-hoc", mostly with the concern for experimental control. While the experimental control is very important, when interpreting the findings and extrapolating the observations to broader tasks and audiences, design of the visualization (that is, the amount of realism, what has been highlighted in the scene, and the location of the highlighted scene elements) should be a part of the discussion.

Based on these results and our previous work, we surmise that we can use VEs similar to our MixedVE to effectively train especially those in need (older people with lower visuospatial memory and spatial abilities) to learn a route that they will have to take later on in the real world (Lokka, Çöltekin, et al., 2018). Based on what we learned in this study, we hypothesize that real world navigation will be faster after learning one's route in a virtual environment such as our MixedVE in comparison to other VE types. We envision detailed analyses of the individual task types and the sub-tasks in this experiment, and a comparative study that examines virtual-world performance with the realworld performance as future work, among the other open questions listed in this section earlier.

\section{ACKNOWLEDGEMENTS}

This work was supported by the Swiss National Science Foundation (Schweizerischer Nationalfonds zur Förderung der Wissenschaftlichen Forschung) [grant number 200021_149670] (SNSF project VISDOM)

\section{REFERENCES}

Borkin, M. A., Vo, A. A., Bylinskii, Z., Isola, P., Sunkavalli, S. Oliva, A., \& Pfister, H. (2013). What Makes a Visualization Memorable. IEEE Transactions on Visualization and Computer Graphics, 19(12), pp. 2306-2315. http://doi.org/10.1109/TVCG.2013.234

Çöltekin, A., \& Clarke, K. C. (2011). A Representation of Everything. Geospatial Today (Guest Editorial), pp. 26-28.

Çöltekin, A., Francelet, R., Richter, K. F., Thoresen, J., \& Fabrikant, S. I. (2017). The effects of visual realism, spatial abilities, and competition on performance in map-based route learning in men. Cartography and Geographic Information Science. http://doi.org/10.1080/15230406.2017.1344569

Çöltekin, A., Lokka, I. E., \& Zahner, M. (2016). On the Usability and Usefulness of 3D (Geo)Visualizations - A Focus on Virtual Reality Environments. In International Archives of Photogrammetry, Remote Sensing and Spatial Information Sciences, Commission. Prague.

Cowan, N. (1995). Attention and memory: An integrated framework. Oxford Psychology Series (Vol. 26).

Estes, W. K. (2014). Handbook of Learning and Cognitive Processes. In Handbook of Learning and Cognitive Processes (Vol. 5, pp. 271-295). http://doi.org/10.4324/9781315770314

Lokka, I. E., \& Çöltekin, A. (2017). Toward optimizing the design of virtual environments for route learning: empirically assessing the effects of changing levels of realism on memory. International Journal of Digital Earth, 8947 pp. 1-19. http://doi.org/10.1080/17538947.2017.1349842

Lokka, I. E., Çöltekin, A., Wiener, J., Fabrikant, S. I., \& Röcke, 
C. (2018). Virtual environments as memory training devices in navigational tasks for older adults. Scientific Reports.

http://doi.org/ 10.1038/s41598-018-29029-x

Park, D. C., Lautenschlager, G., Hedden, T., Davidson, N. S., Smith, A. D., \& Smith, P. K. (2002). Models of visuospatial and verbal memory across the adult life span. Psychology and Aging, 17(2), pp. 299-320. http://doi.org/10.1037/08827974.17.2.299

Röser, F., Hamburger, K., Krumnack, A., \& Knauff, M. (2012). The Structural Salience of Landmarks: Results from an On-Line Study and a Virtual Environment Experiment. Journal of Spatial Science, 57(1), pp. 37-50.

http://doi.org/10.1080/14498596.2012.686362

Sekuler, R., Kahana, M. J., McLaughlin, C., Golomb, J., \& Wingfield, A. (2005). Preservation of Episodic Visual

Recognition Memory in Aging. Experimental Aging Research, 31(1), pp. 1-13. http://doi.org/10.1080/03610730590882800
Smallman, H. S., \& Cook, M. B. (2011a). Naive Realism: Folk fallacies in the Design and Use of Visual Displays. Topics in Cognitive Science, 3(3), pp. 579-608. http://doi.org/10.1111/j.1756-8765.2010.01114.x

Smallman, H. S., \& Cook, M. B. (2011b). Naïve Realism: Folk Fallacies in the Design and Use of Visual Displays. Topics in Cognitive Science, 3(3), pp. 579-608. http://doi.org/10.1111/j.1756-8765.2010.01114.x

Thoresen, J. C., Francelet, R., Coltekin, A., Richter, K. F., Fabrikant, S. I., \& Sandi, C. (2016). Not all anxious individuals get lost: Trait anxiety and mental rotation ability interact to explain performance in map-based route learning in men. Neurobiology of Learning and Memory, 132, pp. 1-8. http://doi.org/10.1016/j.nlm.2016.04.008 